\title{
Distribution Optimal Power Flow
}

\author{
H. M. Khodr, Member, IEEE, M. A. Matos, Member, IEEE, and J. Pereira
}

\begin{abstract}
This paper presents a new and efficient methodology for network reconfiguration with optimal power flow based on Benders Decomposition approach. The objective minimizes the power losses, balancing load among the feeders and subject to the constraints: capacity limit of the branches, minimal and maximal limits of the substation or generator, minimum deviation of the nodes voltages and radial operation of the networks.

A variant of the generalized Benders decomposition algorithm is applied for solving the problem, since the formulation can be embedded under two stages. The first one is the Master problem and is formulated as Mixed Integer non-Linear Programming. This stage determines the radial topology of the distribution network. The second stage is the Slave problem and is formulated as a non-Linear Programming problem. This stage is used to determine the feasibility of the Master problem solution by means of an Optimal Power Flow and provides information to formulate the linear Benders cuts.

The model is programmed in GAMS mathematical modeling language. The effectiveness of the proposal is demonstrated through an example extracted from the specialized literature.
\end{abstract}

Index Terms-Benders Decomposition, Distribution System, Optimal Power Flow, Optimization, Optimal Reconfiguration

\section{NOMENCLATURE}

$N_{d} \quad$ Number of the load nodes of the network

$K_{l} \quad$ Number of lines that enters at node i.

$C_{k}^{i} \quad$ Losses coefficient cost due to the power that circulates at line $\mathrm{k}$

$C_{k}^{i} \quad[\$ /(\mathrm{kVA}$. year $)]$

$K_{\text {OUT }} \quad$ Set of lines that leave node.

$K_{I N} \quad$ Set of lines that enter at node

$K_{2}(i) \quad$ Number of lines that leave node $i$.

$S_{i} \quad$ Load connected to node $i(\mathrm{kVA})$.

$S_{k}^{i} \quad$ Apparent power of line $k$ that enters to node $i$ in kVA.

$S_{l}^{i} \quad$ Apparent power of line $l$ that leaves node i in kVA.

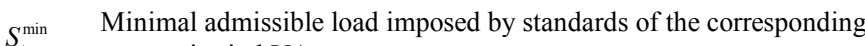
$S_{t} \quad$ companies in kVA.

$N_{C P} \quad$ Number of transformers capacity installed in the point $\mathrm{p}$.

$S^{\max } \quad$ Maximum demands established at lines.

$w_{k}^{i} \quad$ Decision variable to install line $k$ in the node $i$.

I Reference number of the node.

$K_{l} \quad$ Reference number of the line.

This work was supported by Fundação para a Ciência e a Tecnologia Portugal Grant FCT-SFRH/BPD/21035/ 2004.

H.M. Khodr is with INESC Porto, Campus FEUP, Rua Dr. Roberto Frias, 378, 4200 - 465 Porto, Portugal Portugal (e-mail: hkhodr@inescporto.pt).

M. A. Matos is with INESC Porto and the Faculdade de Engenharia da Universidade do Porto, Portugal (e-mail: mmatos@inescporto.pt).

J. Pereira is with INESC Porto and the Faculty of Economics of the University of Porto, Portugal (e-mail: jpereira@inescporto.pt).
$N_{t} \quad$ Number of the transformation point.

$S_{k}^{p} \quad$ Apparent power circulates in the line k that leaves the transformation point $\mathrm{p}$.

$S_{t}^{\max } \quad$ Maximal power apparent of each transformation point in KVA

$y_{t}^{p} \quad$ Decision variable to install the transformer $\mathrm{t}$ en the point $\mathrm{p}$.

$P_{t}^{p} \quad$ Real power output of generating unit or transformer $t$ at bus $p$.

$P_{t}^{\max } \quad$ Maximum real power output of generation unit or transformer $t$

$P_{t}^{\min } \quad$ Minimum real power output of generation unit $t$.

$Q_{t}^{p} \quad$ Reactive output of generating unit or transformer $t$ at bus $p$

$Q_{t}^{\max } \quad$ Maximum reactive output of generation unit or transformer $t$

$Q_{t}^{\min } \quad$ Minimum real power output of generation unit $t$.

$B_{r, n}^{s h} \quad$ Susceptance of the capacitor or reactor $r$ connected at bus $n$.

$Q_{r}^{n} \quad$ Reactive output of capacitor or reactor $r$ connected at bus $n$.

$V_{n} \quad$ Bus voltage magnitude at bus $n$.

$\delta_{n} \quad$ Phase angle at bus.

$G_{j n} \quad$ Real term of the element $j, n$ in bus admittance matrix.

$B_{j n} \quad$ Imaginary term of the element $j, n$ in bus admittance matrix.

$P_{j}^{g} \quad$ Real power injected by all the generating units and transformers connected at bus $j$.

$P_{j}^{\mathrm{d}} \quad$ Real load demand at bus $j$.

$Q_{j}^{g} \quad$ Reactive power injected by all the generating units and transformers connected at bus $j$.

$Q_{j}^{\mathrm{d}} \quad$ Reactive load demand at bus $j$.

$\Phi_{n} \quad$ Subset of all system branches connected at bus $n$.

$V_{n} \quad$ Bus voltage at bus $n$.

$y_{j n} \quad$ Line series admittance.

$\mathrm{y}_{j n}^{\prime} \quad$ Charging admittance.

$V^{\min } \quad$ Minimum voltage at any node $n$.

$V^{\max } \quad$ Maximum voltage at any node $n$.

$R T_{j n}^{m i n} \quad$ Minimum tap of transformer $(j n)$.

$R T_{j n}^{\max } \quad$ Maximum tap of transformer $(j n)$.

$r t_{n j} \quad$ Continuous variable that represents the tap value for transformer $(n j)$

$\Phi_{n}^{R T} \quad$ Subset of all transformers connected at bus $n$.

Dual variable supplied by the slave sub-problem in each iteration $m$, $\lambda_{t, p}^{m} \quad$ which is associated to the decision of connection, the transformer or generator $t$.

$\mu_{l,}^{m} \quad$ Dual variable supplied by the slave subproblem in each iteration $m$,

$\mu_{k, i}^{m} \quad$ which is associated to the decision of installation of the line $k$ at bus $i$.

$Y_{t}^{p, m-1} \quad$ Status of connection (1/0) of the transformer or generator $t$ in the previous iteration $m-1$.

$W_{k}^{i, m-1}$ Status of connection (1/0) of the line $k$ in the previous iteration $m-1$.

\section{INTRODUCTION}

$\mathrm{O}$ PTIMAL Power Flow (OPF) has been extensively used in power systems at the generation/transmission level to 
designate the problem of finding the optimal value for the control variables (real and reactive power, voltage settings, batteries set points, etc.) when minimizing the total operation cost, while respecting the technical constraints of the network and equipments. In distribution systems, many approaches exist dedicated to the optimization of the configuration and the optimization of the voltage profile through batteries. In both cases the objective function seeks for losses reduction, but in separate exercises. On the other hand, the recent development of distribution systems has led to the presence of distributed generation that introduces uncertainty in the previously mentioned problems, but may contribute to voltage control and optimization. In the same cases, regional dispatch of this unit is possible, turning it possible to seek for optimal operating policies.

This paper introduces the new concept of Distribution Optimal Power Flow (DOPF), while aims at integrating all the decision problems mentioned before in a unified model where reconfiguration, distributed generation and batteries are used to find the optimal operating point for the distribution network.

Distribution networks are normally designed meshed but the operation is always configured radially. Their configurations may be varied manual or automatic switching operation for the loads supplied at the minimal cost of possible line losses, increasing system security and enhancing power quality [1]. Reconfiguration consists of the change of the network configuration by means of opening/closing of sectionalizing and tie-switches so that the networks become radial in operation.

Several researches has been conducted for loss minimization in the area of network reconfiguration of distribution systems [3-8], voltage control but few authors have jointly tried the theme of reconfiguration with optimal power flow, in an only joint model at the same time.

Distribution system reconfiguration for loss reduction was first proposed by Merlin and Back [4]. They used the Branch and Bound optimization technique to determine the radial configuration with minimal losses. In this method, all the switches are firstly closed to form the meshed network. The switches are opened successively to restore a radial configuration via destructive method. Shirmohammadi and Hong [5] have suggested a heuristic algorithm based on Merlin and Back algorithm [4]. Here also, the solution procedure starts by closing all the network switches which are opened one after other so as to establish the optimum flow pattern in the networks using many approximations. Borozan et al. [6] have presented a method similar to [5]. However, this method contains three main parts: load estimation, effective determination of power losses configuration and cost/benefit evaluation. Civanlar et al. [7] made use of heuristics and Branch Exchange technique to determine a distribution system configuration, which would reduce line losses. Baran and $\mathrm{Wu}$ [8] have made an attempt to improve the method of Civanlar et al. [5] by introducing two approximation formulas for power flow in the transfer of system loads. Kashem et al. [9] have proposed a branch exchange method for network reconfiguration. This is basically an extensive search method and need to consider all the tie switches. Chen and Cho [10] have performed an analysis of an hourly reconfiguration schedule. They have studied the hourly load patterns over an interval of a year in order to define the hourly load conditions for each season. They have used branch and bound technique for obtaining minimum losses configuration. Nara et al. [11] have proposed a method of distribution system reconfiguration for reduction of real power losses using genetic algorithm. Lin et al. [12] have applied refined genetic algorithm to network reconfiguration problem for reduction of resistive line losses. Huang [13] has proposed one genetic algorithm based on fuzzy approach for network reconfiguration of distribution system. Although the researchers [11-13] have demonstrated the effectiveness of genetic algorithm for network reconfiguration, solution time is highly prohibitive. Lin and Chin [14-15] have presented an algorithm for distribution feeder reconfiguration. They have used voltage index, ohmic index and decision index to determine the switching operation. Huang and Chin [16] have proposed an algorithm based on fuzzy operation to deal with the feeders reconfiguration problem. Their approach tries to minimize power losses and acquire the load balance at the same time. Liu et al. [17], Jung et al. [18] and Auguliaro et al. [19] have proposed applications based on artificial intelligence for a minimum losses configuration. Hsiao [20] has proposed fuzzy multi-objective based evolution programming method for network reconfiguration.

Matos et al. [32-34] present an approach to the reconfiguration of radial distribution networks, for both loss reduction and service restoration using the simulated annealing meta-heuristic technique, in this works the authors minimize the loss or load not supplied, the total number of switching operations is included as a possible criterion. In [33] the same authors consider a set of load scenarios and finding single or multiple configuration solutions that minimize both energy losses and switching actions, in a bi-criteria framework, using the same meta-heuristic technique.

Pereira et al [35] presents a model to identify optimal operation strategies of electric distribution networks minimizing the active power losses taking into account the transformers taps and the capacitor banks that are in operation. This problem is solved by meta-heuristic technique Simulated Annealing and tested in a realistic problem based on a Portuguese distribution system.

It can be appreciated that the reconfiguration systems with optimal power has not been presented or studied at the same time and in the same model.

The distribution reconfiguration and the OPF problems, has been addressed and studied separately by many works reported in the literature. [1-20] are dedicated to the reconfiguration problem and [21-26] are dedicated to OPF.

In [36] presents a new approach for distribution system reconfiguration (DSR) based on OPF in which the branch statuses (open/close) are presented by continuous functions. In the approach all branches are initially considered closed, and from the OPF results, a heuristic technique is used to determine the next loop to be broken by opened one switch. Then the list of switches that are candidates to be opened is 
updated, and the above process is repeated until all loops are broken, making the distribution system radial.

The formulations differ from each other due to the representation in the model of more or fewer problem characteristics and/or the use of different solution algorithms. The problem is such that more characteristics are modeled the higher is the required computational effort. Most of the authors consider the formulation problem in two separate problems; the first one consisting of the optimal reconfiguration of distribution networks using divers' method and the second is the OPF. In this work, we consider the two problems as the same one. This problem is solved by Benders decomposition algorithm.

This paper aims to expand the previous works by including and solving the Master problem with implicit enumeration algorithm, and to address the objective of the distribution networks Optimal Reconfiguration. It is shown that feasibility seeking variant of the Benders algorithm using implicit enumeration is very efficient solving reconfiguration and optimal power flow of large-scale distribution systems with very short computational time.

This paper is structured as follows. Section 2 deals with optimal networks reconfiguration problem, and defines the model equations. Section 3 briefly describes the method applied to solve the problem, the Generalized Benders decomposition, defining the Master problem as mixed-integer linear programming, and the Slave problem as non-linear programming. In both cases the set of constraints and the objective function are listed. Likewise the problem of the Benders algorithm feasibility is described. In section 4, the solution procedure for the NLP subproblem and master problem are detailed, and illustrates the most of the proposed model facilities and potentialities in the case study extracted to [1]. Finally, Section 5 states the conclusions.

\section{PROBLEM FORMULATION}

The DOPF model must be able to determine the optimal configuration, and evaluate the load distribution amongst substations and feeders. The resulting system should be feasible, i.e. it meets demand and technical requirements. There is a considerable amount of feasible alternatives and the model chooses the alternative that optimizes a specific objective function.

The single objective formulation of the DOPF has already been commented in the previous section. An objective function, including the minimization of the losses, has been considered. The set of constraints comprises: power balance constraints - 1st Kirchhoff's law; capacity limits of the existing substations and feeders; voltage calculation constraints - 2nd Kirchhoff's law; voltage limits for all load buses; radiality constraints; logical constraints - involving integer decision variables - and all the OPF constraints. The DOPF formulation with objective function and set of constraints is stated as follows:

Cost of the power losses:

$$
Z=\sum_{i \in N_{d}} \sum_{k \in K_{l}} C_{k}^{i} S_{k}^{i^{2}}
$$

$C_{k}^{i}=C 1_{k}^{i}$ if branch is three-phase and $C_{k}^{i}=C 3_{k}^{i}$ if branch is mono-phase three wires.

First Kirchhoff law in the nodes or power balance in the nodes:

$\sum_{k \in K_{I N}} S_{k}^{i}-\sum_{l \in K_{\text {OUT }}} S_{l}^{i}=S_{i} ; \quad \forall i \in N_{d}$

Minimal power output limits of the substation or generator:

$\sum_{k \in K_{O U T}} S_{k}^{p} \geq \sum_{t \in N_{C P}} S_{t}^{\min } \cdot y_{t}^{p} ; \quad \forall p \in N_{t}$

Maximal power output limits of the substation or generator $\sum_{k \in K_{\text {OUT }}} S_{k}^{p} \leq \sum_{t \in N_{C P}} S_{t}^{\max } \cdot y_{t}^{p} ; \quad \forall p \in N_{t}$

Power limits of the Lines or thermal limits of the Lines:

$S_{k}^{i} \leq S_{k, i}^{\max } \cdot w_{k}^{i} ; \quad \forall k \in K_{l}, \forall i \in N_{d}$

Radial operation condition of the networks:

$\sum_{k \in K_{I N}} w_{k}^{i} \leq 1 ; \quad \forall i \in N_{d}$

Between tow nodes, the line is represented by double direction of the power flow. In the operation of the networks only one of the directions should be exist.

If the line $l k=I T(i, k) ; k<k l(i)$ and $k l=I N(i, k) ; k<k 2(i)$

Then: $j=1, \cdots, N d$

General power balance in the networks: $\sum_{p \in N_{t}} \sum_{k \in K_{\text {OUT }}} S_{k}^{p} \leq \sum_{p \in N_{t}} \sum_{t \in N_{C P}} S_{t}^{\max } \cdot y_{t}^{p}$

Real power output limits:

$y_{t}^{p} \cdot \mathrm{P}_{t}^{\min } \leq P_{t}^{p} \leq \mathrm{P}_{t}^{\max } \cdot y_{t}^{p} ; \forall t \in N_{C P}, \forall p \in N_{t}$

Reactive power output limits:

$y_{t}^{p} \cdot \mathrm{Q}_{t}^{\min } \leq Q_{t}^{p} \leq \mathrm{Q}_{t}^{\max } \cdot y_{t}^{p} ; \quad \forall t \in N_{C P}, \forall p \in N_{t}$

Reactive power output by shunt capacitors:

$Q_{r}^{n}=\mathrm{B}_{r, n}^{\mathrm{sh}}\left(V_{n}\right)^{2} ; \forall r \in N_{c}, \forall n \in N_{d}$

Real power flow equations:

$\sum_{n \in N_{d}} V_{j} \cdot V_{n} \cdot\left(G_{j n} \cdot \cos \left(\delta_{j}-\delta_{n}\right)+B_{j n} \cdot \sin \left(\delta_{j}-\delta_{n}\right)\right)=P_{j}^{\mathrm{g}}-\mathrm{P}_{\mathrm{j}}^{\mathrm{d}}$;

$\forall j \in N_{d}: P_{j}^{g}=\sum_{t \in N_{C P}} P_{t}^{j}$

Reactive power flow equations:

$\sum_{n \in N_{d}} V_{j} \cdot V_{n} \cdot\left(G_{j n} \cdot \sin \left(\delta_{j}-\delta_{n}\right)-B_{j n} \cdot \cos \left(\delta_{j}-\delta_{n}\right)\right)=Q_{j}^{g}-Q_{j}^{\mathrm{d}} ;$

$\forall j \in N_{d}: Q_{j}^{\mathrm{g}}=\sum_{t \in N_{C P}} Q_{t}^{j}$

Distribution capacity limits:

$\left|V_{j} \cdot\left[\left(V_{j}-V_{n}\right) \cdot y_{j n}\right]^{*}+V_{j} \cdot\left(V_{j} \cdot\left(\frac{1}{2} \cdot y_{j n}^{\prime}\right)^{*}\right)\right| \leq S_{j, n}^{\max } ;$

$\forall j, n \in N_{d}:(j n) \in \Phi_{j}$

\section{A. Benders Decomposition}

The DOPF problem addressed in this paper is a mixedinteger non-linear optimization problem with non-linear objective function, binary decision variables, continuous variables for operation processes, and non-linear constraints such as complete power flow equations or transmission capacity limits.

The difficulties related to resolution of non-linear optimization problems with binary variables force to make use 
of partitioning techniques as Benders decomposition $[1,2]$. The Benders partition algorithm is a decomposition technique in two-levels, master and slave, which defines an iterative procedure between both levels in order to reach the optimal solution. The master level represents the decision problem, mixed-integer non-linear optimization problem, whereas the slave level deals with the operation problem, non-linear OPF. This method allows to treat appropriately the non-convexity associated to binary variables and to divide the global problem into two smaller problems easier to solve.

The master problem determines the new configuration of the network and the opened switches. This solution is transferred to the slave sub-problem, which verifies the feasibility of the master problem solution and the dual values associated to the configuration decision taken previously by the master problem. In the iterative process, this information is supplied in the next iteration to the master problem through the Benders cuts in order to improve the new decision of the master problem.

The procedure followed in this paper includes the steps illustrated in the flowchart of the Fig. 1. The "start" point will be the initial configuration of the network.

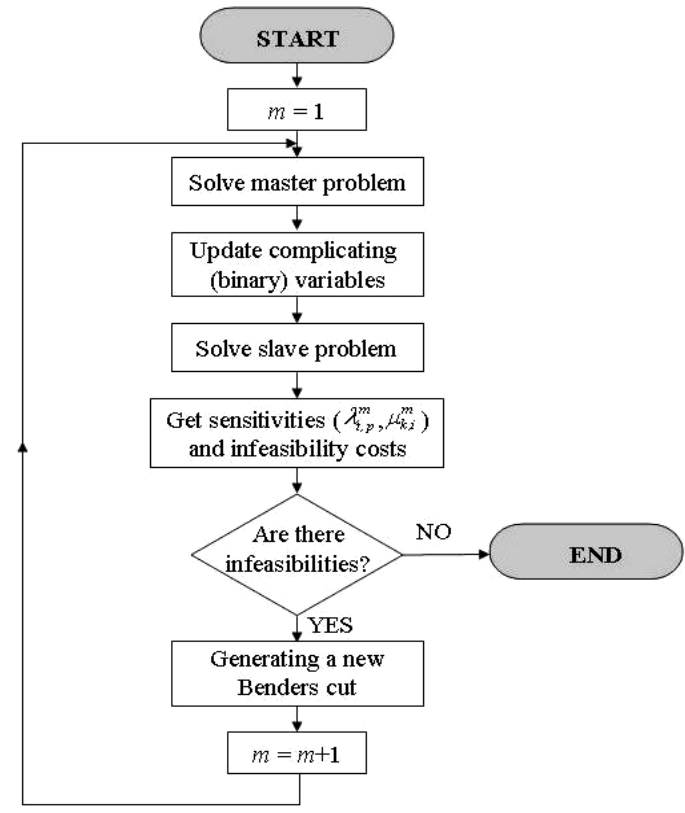

Fig. 1 Benders decomposition flowchart

\section{Master Problem}

The master problem decides the network configuration in order to minimize the system power losses by opening/closing tie-switches ( $1 / 0$ binary variables) so that the network becomes radial in operation. Therefore, all binary variables have to be included in the optimization problem of this level.

The objective function minimizes:

$\sum_{i \in N_{d}} \sum_{k \in K_{l}} C_{k}^{i} S_{k}^{i}+\alpha^{*}$

Subject to the constraints (2)-(7) and the Benders cuts:

$$
\begin{aligned}
\alpha^{*} & \geq \alpha\left(Y_{t}^{p, m-1}, W_{i}^{k, m-1}\right)+\sum_{p \in N_{t}} \sum_{t \in N_{C P}} \lambda_{t, p}^{m-1} \cdot\left(y_{t}^{p, m-1}-Y_{t}^{p, m-1}\right)+ \\
& +\sum_{k \in K_{l}} \sum_{i \in N_{d}} \mu_{k, i}^{m-1} \cdot\left(w_{k}^{j, m-1}-W_{k}^{i, m-1}\right) \quad \forall m \in M
\end{aligned}
$$

where $\alpha\left(Y_{t}^{p, m-1}, W_{i}^{k, m-1}\right)$ is the slave problem cost in the iteration $\mathrm{m}-1$.

The first term of the objective function represents the operation cost (power losses cost). The second term means, through the real variable $\alpha^{*}$, an underestimation of the slave subproblem costs or feasibility costs. Therefore, the optimization variables of this problem are $\alpha^{*}, S_{k}^{i}$.

The key issue in Benders decomposition is located at equations (15), the named Benders linear cuts, which are feasibility cuts in this problem. The levels, master and slave, are coupled by these cuts which are updated at each iteration.

\section{Slave Subproblem}

The slave level checks the feasibility of the master problem solution and provides the optimal value of the operation variables by means of an AC OPF. Therefore, the slave subproblem solves system operation, minimizing the cost of infeasibility, as there could be some cases where the master level solution does not guarantee its feasibility. It would force to add slack values at some buses (bus voltage violation) or at some lines (lines overload) and to include them in the objective function of the slave problem so that the objective function minimizes the infeasibilities of the system. The end solution of the global problem has to be feasible and optimal, that is, all the slack variables should be equal to zero.

The objective function would be formulated as:

$\sum_{n \in N} P_{n}^{f i c}+Q_{n}^{f i c L}+Q_{n}^{f i c C}+S_{n}^{-f i c}$

subject to the constraints (8)-(13) and:

$y_{t}^{p}=Y_{t}^{p, m} \quad: \lambda_{t, p}^{m-1} \quad \forall t \in N_{C P}, \forall p \in N_{t}$

$w_{k}^{i}=W_{k, i}^{i, m} \quad: \mu_{k, i}^{m-1} \quad \forall k \in K_{l}, \forall i \in N_{d}$

The minimization is subject to the AC power flow equations, real and reactive output production limits, transmission capacity limits of lines, voltage magnitude and bus angle limits, Therefore, the slave problem determines the values of the operation variables $\left(P_{t, i}, Q_{t, k}, V_{t, n}, \delta_{t, n}, r_{t, n j}\right)$ at each period for all operating states. Besides, the equation (17) supplies the sensitivity for each value of the decision variables $\left(y_{t}^{p}, w_{k}^{i}\right)$ fixed by the master problem at the same iteration.

The slack variables $P_{n}^{f i c}, Q_{n}^{f i c L}, S_{n}^{f i c}$ would be included in the equations (11), (12) and (13); which would be formulated as:

$\sum_{n \in N_{d}} V_{j} \cdot V_{n} \cdot\left(G_{j n} \cdot \cos \left(\delta_{j}-\delta_{n}\right)+B_{j n} \cdot \sin \left(\delta_{j}-\delta_{n}\right)\right)=$

$=P_{j}^{g}+P_{n}^{f i c}-\mathrm{P}_{\mathrm{j}}^{\mathrm{d}} ; \quad \forall j \in N_{d}: P_{j}^{g}=\sum_{t \in N_{C P}} P_{t}^{j}$

$\sum_{n \in N_{d}} V_{j} \cdot V_{n} \cdot\left(G_{j n} \cdot \sin \left(\delta_{j}-\delta_{n}\right)-B_{j n} \cdot \cos \left(\delta_{j}-\delta_{n}\right)\right)=$

$=Q_{j}^{g}+Q_{n}^{\text {ficc }}-Q_{n}^{\text {ficl }}-Q_{j}^{\mathrm{d}} ; \quad \forall N_{j} \in N_{d}: Q_{j}^{g}=\sum_{t \in N_{C P}} Q_{t}^{j}$

$\left|\mathbf{V}_{j} \cdot\left[\left(\mathbf{V}_{j}-\mathbf{V}_{n}\right) \cdot \mathbf{y}_{j n}\right]^{*}+\mathbf{V}_{j} \cdot\left(\mathbf{V}_{j} \cdot\left(\frac{1}{2} \cdot \mathrm{y}_{j n}^{\prime}\right)^{*}\right)\right|$

$-S_{n}^{f i c} \leq \mathrm{S}_{j n}^{\max } ; \quad \forall j, n \in N_{d}: \quad(j n) \in \Phi_{j}$

B. Benders convergence criterion

Benders decomposition procedure stops when the provided solution by the master problem is feasible. In this case, the value of the objective function computed in the slave problem 
is equals the master problem cost, except for a small cost tolerance.

\section{Test Case}

The tested system is a $11 \mathrm{kV}$ radial distribution system having two substations, four feeders, 70 nodes and 78 branches (including tie-branches) and all data for this system are extracted from [1] of the Appendix A.

The formulation of the optimization problem is based on the network of the fig. 2. This figure represents the distribution network to reconfigure, where the power flow is presented in an only direction if this circulates since the source toward the load nodes. When a doubt to feed the load nodes since any substation exist, in this case this is represented with a line with double power flow sense, to give him all the possibilities to each node to be fed since any substation. The proposed method belongs to the destructive methods. The mathematical model eliminates the lines in each iteration. The stop criterion is if is eliminated a line remains a load without feeding, this condition is guaranteed by means of the model constraints.

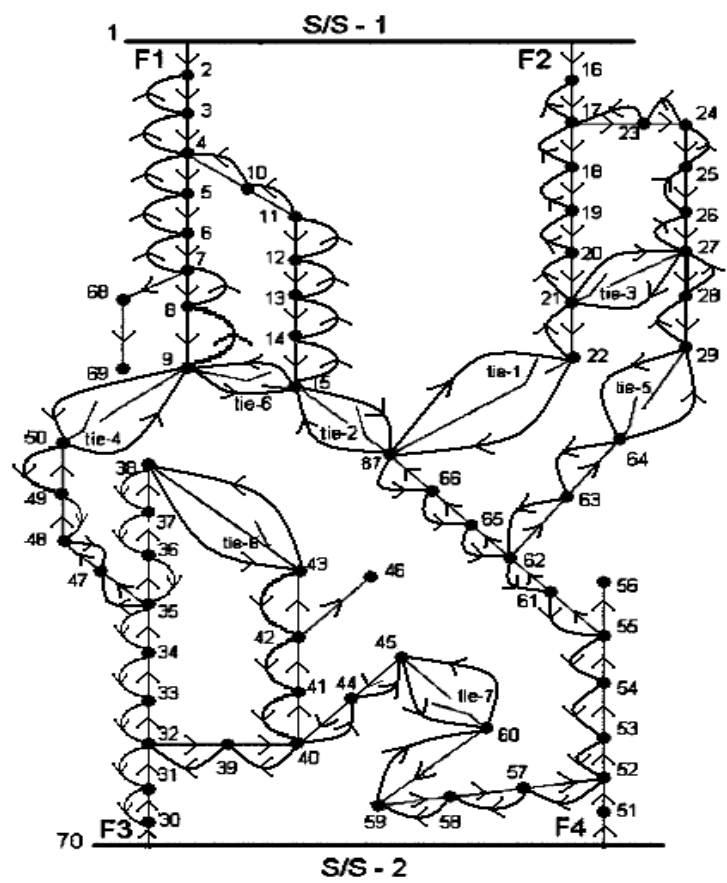

Fig. 2. Representation of the distribution network

In [1] have mentioned that before network reconfiguration, the total real power loss of this system is $337.45 \mathrm{~kW}$ and the minimum voltage is $V_{\text {min }}=V_{67}=0.88389$ p.u.

After the application of the methodology exposed in [1], the real power losses is reduced to $302.05 \mathrm{~kW}$ and the minimum voltage is elevated to $V_{\min }=V_{29}=0.91214$ p.u.

After the application of the proposed methodology the real power losses of this systems is $268.55 \mathrm{~kW}$ and the minimum voltage is $V_{\text {min }}=V_{64}=0.93418 p . u$. In the Fig. 3 shows the final radial configuration after the application of the proposed methodology.

The comparison between the proposed methodology and the methodology exposed in the reference [1] is presented in the Table I.

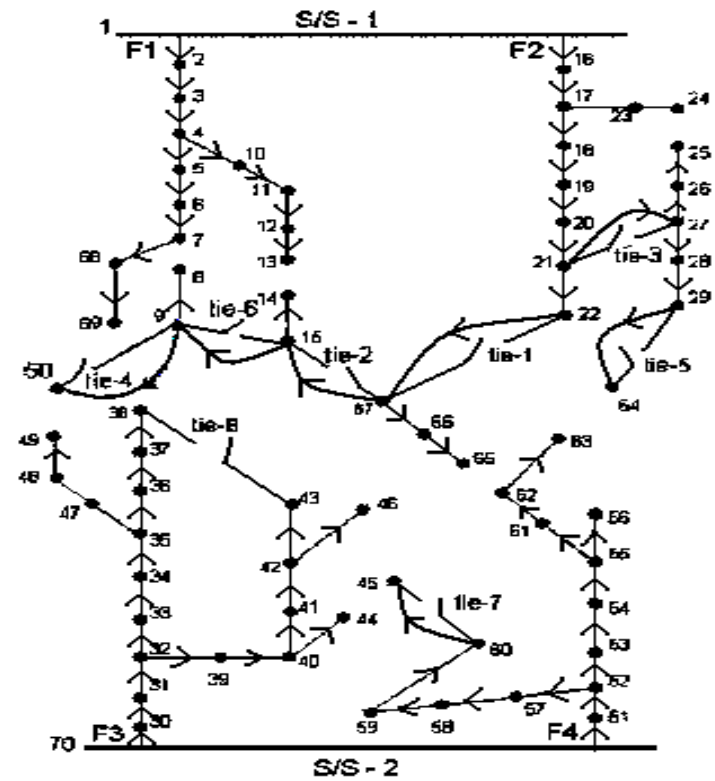

Fig. 3. Final radial configuration system obtained by the proposed methodology

TABLE I

COMPARISON BETWEEN THE PROPOSED METHODOLOGYAND THE METHODOGY EXPOSED IN THE REFERENCE [1]

\begin{tabular}{ccc}
\multicolumn{3}{c}{ EXPOSED IN THE REFERENCE [1] } \\
\hline \multicolumn{3}{c}{ Proposed Methodology $\begin{array}{c}\text { Methodology Exposed } \\
\text { in the Reference [1] }\end{array}$} \\
\hline $\begin{array}{c}\text { Real Power Losses in the } \\
\text { Actual State of the Networks } \\
\text { before reconfiguration }\end{array}$ & $337.45 \mathrm{~kW}$ \\
\hline $\begin{array}{c}\text { Real Power Losses after } \\
\text { reconfiguration }\end{array}$ & $288.55 \mathrm{~kW}$ & $302.05 \mathrm{~kW}$ \\
\hline $\begin{array}{c}\text { Reduction of Power Losses in } \\
\% \text { after reconfiguration }\end{array}$ & $14.49 \%$ & $10.49 \%$ \\
\hline $\begin{array}{c}\text { Minimum Voltage before } \\
\text { reconfiguration }\end{array}$ & $V_{\min }=V_{67}=0.88389 \mathrm{p.u}$. \\
\hline $\begin{array}{c}\text { Minimum Voltage after } \\
\text { reconfiguration }\end{array}$ & $V_{\min }=V_{64}=0.93418 p . u . \quad V_{\min }=V_{29}=0.91214 p . u$. \\
\hline
\end{tabular}

To prove the proposed methodology, the database that have extracted from [1] has been modified, where have been settled two batteries of condensers in the nodes 49 and 64 with three steps each one: 150,300 and 600 kvar. The tap's of the transformers has been considered in the two substations, 1 and 70 so that the tension varied between 0.96 and 1.04 per unit (p.u.). Two generators of 2 MVA each one has been installed in the nodes 15 and 29. The execution of the proposed methodology has obtained the following results:

The tap of the transformer connected in the node 1 not changed, but in the node 70 the transformer tap is adjusted to maintain the node voltage upper the 0.96 p.u. In this case the tap should be elevated in 0.038 p.u.

Step two of 300 kvar of the capacitors batteries has been selected by the methodology. As for the generators when considering the operation costs of these didn't install, since it is more economic the operation of the net without them. The active power losses decreased in $3.2 \%$. The nodes voltages of the network are between 0.96 p.u. and 1.04 p.u. The iterations number is of 570 and the execution time of the methodology is of $0.45 \mathrm{Sec}$. in a computer with a Intel Core 2 Duo Processor T7200 and 2 GB of RAM. 


\section{CONCLUSION}

In this paper was presented a new and efficient methodology for DOPF based on Benders Decomposition approach. The objective minimizes the power losses balancing load among the feeders and subject to the constraints: capacity limit of the branches, minimal and maximal limits of the substation, minimum deviation of the nodes voltages and radial operation of the network.

A variant of the generalized Benders decomposition algorithm was applied for solving the problem, since the formulation can be embedded under two stages. The first one is the Master problem and is formulated as Mixed Integer Quadratic Programming. This stage determines the radial topology of the distribution network. The second stage is the Slave problem and is formulated as a non-Linear Programming problem. This stage is used to determine the feasibility of the Master problem solution by means of an OPF and provides information to formulate the linear Benders cuts.

The application of the methodology to the modified example extracted from literature, demonstrates the effectiveness, and the robustness of the proposal. It is also verified that have a very low execution time for solving a Mixed Integer Quadratic Programming and also a non-Linear Programming problem.

\section{REFERENCES}

[1] D. Das, "Reconfiguration of distribution system using fuzzy multiobjective approach", Electrical Power and Energy Systems 28 (2006) 331-338, Aug. 2005

[2] N. Kagan, R.N. Adams, "A Benders' decomposition to multi-objective distribution planning problem", International Journal of Electrical Power and Energy Systems, Vol. 15, Issue 5, 1993, pp. 259-271.

[3] R.J. Sarfi, M. M.A. Salama, A.Y. Chikhani, "A survey of the state of the art in distribution system reconfiguration for system loss reduction", Electric Power Systems Research 1994; 31:61-70.

[4] A. Merlin, H. Back, "Search for a minimal-loss operating spanning tree configuration in an urban power distribution system", Proceedings of the fifth power system computation conference (PSCC), Cambridge, UK, 1975, pp.1-18.

[5] D. Shirmohammadi, H.W. Hong, "Reconfiguration for electric distribution networks for resistive line loss reduction", IEEE Trans. Power Delivery 1989; 4:1910-8.

[6] V. Borozan, D. Rajicic, R. Ackovski, "Improved method for loss minimization in distribution networks", IEEE Trans Power Systems 1995; 10: 1420-5.

[7] S. Civanlar, J.J. Grainger, H. Yin, S.S.H. Lee, "Distribution feeder reconfiguration for loss reduction", IEEE Trans. Power Delivery 1988; 3: 1217-23.

[8] M.E. Baran, F.F. Wu, "Network reconfiguration in distribution systems for loss reduction and load balancing", IEEE Trans. Power Delivery 1989;4:1401-7.

[9] M.A. Kashem, V. Ganapathy G.B. Jasmon., "Network reconfiguration for enhancement of voltage stability in distribution networks", IEE Proc. Gener. Transm. Distrib., 2000; 147(3):171-5.

[10] C.S. Chen, M.Y. Cho, "Energy loss reduction by critical switches", IEEE Trans. Power Delivery 1993; 8:1246-53.

[11] K. Nara, T. Satoh, M. Kitagawa, "Distribution system loss minimum reconfiguration by genetic algorithm", Proceedings of the third symposium expert systems application to power systems (ESAPS), Tokyo and Kobe, Japan; 1991. p. 724-30.

[12] W.M. Lin, F.S. Cheng, M.T. Tsay, "Distribution feeder reconfiguration with refined genetic algorithm", IEE Proc. Gener. Transm. Distrib. 2000; 147(6): 349-54.
[13] Y.C. Huang, "Enhanced genetic algorithm-based fuzzy multi-objective approach to distribution network reconfiguration", IEE Proc. Gener. Transm. Distrib 2002; 149(5):615-20.

[14] W.M. Lin, H.C. Chin, "A new approach for distribution feeder reconfiguration for loss reduction and service restoration", IEEE Trans. Power Delivery, 1998; 13:870-5.

[15] W.M. Lin, H.C. Chin, G.J. Yu, "An effective algorithm for distribution feeder loss reduction", IEEE International Conference of Power System Technology 1998; 1:228-32.

[16] K.Y. Huang, H.C. Chin, "Distribution feeder energy conservation by using heuristics fuzzy approach", International Journal of Electrical Power and Energy Systems, 2002; 24: 439-45.

[17] C.C. Liu, S.J. Lee, S.S. Venkata, "An expert system operational aid for restoration and loss reduction of distribution systems", IEEE Trans. Power Systems, 1988;3:619-26.

[18] K.H. Jung, H. Kim, Y. Ko, "Network reconfiguration algorithm for automated distribution system based on artificial intelligence approach", IEEE Trans. Power Delivery 1993; 8:1933-41.

[19] A. Auguliaro, L. Dusonchet, M. Ippolito, E.R. Sanseverino, "Minimum losses reconfiguration of MV distribution networks through local control of tie switches", IEEE Trans. Power Delivery, 2003; 18:762-71.

[20] Y.T. Hsiao, "Multi-objective evolution programming method for feeder reconfiguration", IEEE Trans. Power Systems 2004; 19:594-600.

[21] Ghosh S, Das D. Method for load-flow solution of radial distribution networks. Proc IEE Gener. Transm. Distrib., 1999; November: 641-8.

[22] H.Y. Yamin, K. Al-Tallaq, S. M. Shahidehpour, "New Approach for Dynamic Optimal Power Flow Using Benders Decomposition in a Deregulated Power Market", Electric Power Systems Research, Vol. 65: pp. 101-107, 2003 .

[23] N. Alguacil, A. J. Conejo, "Multiperiod Optimal Power Flow Using Benders Decomposition", IEEE Trans. on Power Systems, Vol. 15(1): pp.196-201, Feb. 2000.

[24] J.A. Momoh, M. E. El-Hawary, R. Adapa, "A Review of Selected Optimal Power Flow Literature to 1993. Part I: Nonlinear and Quadratic Programming Approaches", IEEE Trans. on Power Systems, Vol. 14(1): pp. 96-104, Feb. 1999.

[25] J.A. Momoh, M. E. El-Hawary , R. Adapa, "A Review of Selected Optimal Power Flow Literature to 1993. Part II: Newton, Linear Programming and Interior Point Methods", IEEE Trans. on Power Systems, Vol. 14(1): pp. 96_104, Feb. 1999.

[26] M. Huneault, F. D. Galiana, "A Survey of the Optimal Power Flow Literature, IEEE Trans. on Power Systems, Vol. 6(2): pp. 762-770, May 1991.

[27] J.F. Benders. "Partitioning Procedures for Solving Mixed-Variables Programming Problems", Numerische Mathematik, Vol. 4: pp. 238-252, 1962.

[28] G. Cohen, "Auxiliary Problem Principle and Decomposition of Optimization Problems", Journal of Optimization Theory and Applications, Vol. 32(3): pp. 277-305, Nov. 1980.

[29] GAMS Development Corporation, Washington, DC 20007, USA. GAMS-The Solver Manuals, GAMS User Notes, Jan. 2001.

[30] A.M. Geofrion, "Generalized Benders Decomposition", Journal of Optimization Theory and Applications, Vol. 10(4): pp. 237-260, 1972.

[31] T.L. Magnanti, R. T. Wong, "Accelerating Benders Decomposition: Algorithmic Enhancement and Model Selection Criteria", Operations Research, Vol. 29(3): pp. 464-484, May-Jun. 1981.

[32] M.A. Matos, P. Melo, "Multi-objective Reconfiguration for Loss Reduction and Service Restoration Using Simulated Annealing", Proc. of IEEE Power Tech'99, Budapest, Hungary, Aug. 1999.

[33] M.A, Matos and P. Melo, "Loss Minimization in Distribution Networks with Multiple Load Scenarios", Proc. of IEEE Porto Power Tech'2001, Vol. 3, Porto Portugal, Sept. 2001.

[34] M.A. Matos et al., "Meta-heuristics Applied to Power Systems", Metaheuristics: Computer Decision-Making, Kluwer Academic Publishers B. V., pp. 449-464, 2003.

[35] J. Pereira et al., "Identification of Operation Strategies of Distribution Networks Using a Simulated Annealing Approach", Proc. of IEEE Power Tech'99, Budapest, Hungary, Aug. 1999.

[36] F. V. Gomes et al., "A New Distribution System Reconfiguration Approach Using Optimum Power Flow and Sensitivity Analysis for Loss Reduction", IEEE Trans. on Power Systems, Vol. 21(4), Nov. 2006. 